\title{
Modelo para incorporar las TIC en las MIPYMES del sector hotelero
}

\author{
Model to incorporate TIC into MIPYMES in the hotel sector
}

\author{
M. M. Arteaga-Martínez iD ; E. M. Esteban-Regino iD ; M. A. Ojeda-Enríquez \\ DOI: https://doi.org/10.22517/23447214.24848 \\ Artículo de investigación científica y tecnológica
}

\begin{abstract}
The research's fundamental purpose was to structure a model to incorporate TIC in the MIPYMES of the hotel sector of the city of Monteria. The research was developed with a mixed approach and an exploratory scope. The validation of the model in three organizations in the hotel sector with basic, intermediate, and advanced use of TIC will check that the competitive benefits resulting from the TIC implemented in said hotels are equivalent or correspond to their characterization's competitive benefits. Although the model to incorporate TIC was conceived to be implemented in the hotel sector of the city of Monteria, it can also be implemented by any hotel regardless of its size and geographical location. It takes into account the processes that are developed in this type of organizations at the local, regional, national and international levels and the technological applications (TIC) proposed to encompass all these processes. The model can be adapted to any other economic sector; for it, the TICs that would make up level 2 control of operational processes and their benefits must be identified since they are different for each sector. The model is aligned with the Ministry of TIC policies MinTIC in the sense that they seek that entrepreneurs in the hotel sector take ownership of the use of technologies to be more competitive.
\end{abstract}

Index Terms - Hotel processes, Model, TIC, TIC appropriation, TIC characterization

Resumen- El propósito fundamental de la investigación fue estructurar un modelo para incorporar las TIC en las MIPYMES del sector hotelero de la ciudad de Montería, la investigación se desarrolló con un enfoque mixto y un alcance exploratorio. La validación del modelo en tres organizaciones del sector hotelero con uso básico, intermedio y avanzado de las TIC permitió cotejar que los beneficios competitivos resultantes de la TIC implementadas en dichos hoteles, equivalen o se corresponden con los beneficios competitivos identificados en la caracterización de estas. Aunque el modelo para incorporar las TIC fue concebido para ser implementado en las organizaciones del sector hotelero de la ciudad de Montería, este puede ser igualmente implementado por cualquier hotel sin importar su tamaño y su ubicación geográfica, dado a que en su estructuración se tuvieron en cuenta los procesos que se desarrollan en este tipo de organizaciones a nivel local, regional, nacional e internacional y las aplicaciones tecnológicas (TIC) propuestas abarcan todos estos procesos. El modelo puede ser adaptado a cualquier otro sector económico, para ello se deben identificar las TIC que conformarían el nivel 2 control de los procesos operacionales y sus beneficios, dado a que son distintas para cada sector. El modelo se alinea con las políticas del Ministerio de las TIC - MinTIC en el sentido que buscan que los empresarios del sector hotelero se apropien del uso de las tecnologías para que sean más competitivos.

Palabras claves - Modelo, TIC, caracterización de las TIC, Procesos hoteleros.

\section{INTRODUCTION}

$\mathrm{D}$ E acuerdo con la primera gran encuesta / 2017 "estudio de acceso, uso y reto de las TIC en Colombia" realizada por MinTIC, solo el 25\% de las empresas forman al talento humano en esta área, por esta razón desde MinTIC se busca que las MIPYMES colombianas hagan una adecuada apropiación de la tecnología para ser cada vez más competitivas a través de iniciativas como la plataforma https://www.empresariodigital.gov.co/, de acuerdo con esta políticas nacional y la importancia de las MIPYMES del sector hotelero para el turismo quien es el segundo generador de divisas en Colombia, la demanda turística en la ciudad de Montería y el diagnóstico de uso de las TIC en el sector hotelero realizado por [1] en el cual se determinó que el nivel de uso de las TIC es del 46\%, razones por las cuales se estructuró el Modelo para incorporar las TIC en las MIPYMES del sector hotelero en la ciudad de Montería, modelo que se detalla en el presente informe.

La investigación se realizó con un enfoque mixto y alcance exploratorio, lo que permitió indagar desde una perspectiva innovadora, cuáles son las TIC utilizadas en las MIPYMES del sector hotelero que contribuyen a su competitividad, para incorporarlas en sus procesos de negocio a través de la estructuración del modelo, que se estructuró a partir de una revisión bibliográfica en la cual se identificaron las TIC que se utilizan en los diferentes procesos de las organizaciones hoteleras y los beneficios competitivos que cada una de estas tributan a dicho sector, posteriormente se caracterizaron en una escala ordinal, teniendo en cuenta diseños previos de incorporación o apropiación de las TIC, lo que permitió definir 
cuatro (4) niveles que conforman el modelo propuesto.

El diseño del modelo se validó teniendo en cuenta el diagnóstico de uso de las TIC realizado por [1], del cual se tomaron tres organizaciones que se encuentren en bajo, medio y alto nivel de uso de las TIC respectivamente, a las cuales se le aplicó un cuestionario con una escala tipo Likert que permitió verificar los beneficios competitivos propuestos por el modelo, finalmente se presentan las conclusiones, discusiones y las referencias bibliográficas.

\section{MARCO TEÓRICO}

\section{A. Antecedentes}

Con el objetivo de establecer un Modelo para incorporar las TIC en las MIPYMES del sector hotelero en la ciudad de Montería, se indagaron antecedentes en el uso de las TIC a nivel internacional, nacional y local. A nivel internacional se tuvieron en cuenta tres antecedentes españoles y uno nicaragüense, el primer antecedente español expone que existe correlación entre el tamaño y la categoría hotelera con la adopción de los sistemas CRM, puesto que estos impactan significativamente en su desempeño económico, maximizando sus beneficios y obteniendo más cuotas del mercado y que además, los hoteles pequeños y medianos carecen de este tipo de herramientas debido a la escasez de recursos, la falta de motivación de los empleados y la insuficiencia de liderazgo de los directivos [3]. El segundo antecedente español, infiere que cuando mayor es la presencia del hotel en Internet, mayor es la intención de sus clientes de generar comentarios en la Red, por lo que son las TIC orientadas al servicio al huésped las que contribuyen en mayor medida a explicar la satisfacción del cliente y sus intenciones de volver a visitar el hotel y recomendar el mismo a través de Internet [4]. Y el tercer antecedente español, revela que el $95 \%$ del sector hoteles, campings y agencias de viajes, le apuestan a mayor equipamiento TIC, en especial a la conectividad con banda ancha móvil y a programas de ecommerce, con los que aprovechan oportunidades de expansión empresarial y ahorro de costes [2]. Por último, en el 1er semestre del 2016, en Estelí - Nicaragua, se estableció que los clientes utilizan internet y organizan su viaje por cuenta propia, el mayor porcentaje de los hoteles cuentan con conexión a internet, computadoras personales para la gestión del negocio, página web corporativa, donde le apuestan al social media y el e-mail marketing gratuito por medio de plataformas web intermediarias de reservas (TripAdvisor, o Booking), aunque la mayoría no cuentan con un plan de marketing definido, entre otras razones porque no están preparados para la venta por internet o no cuentan con software internos y externos que le ayude a la gestión del negocio, reduciéndose a poseer solo un sistema de para la contabilidad [5].

A nivel nacional, una investigación del sector hotelero en la ciudad de Bogotá destaca que existen resultados positivos relacionados con el uso del marketing digital (página web, canales de venta en línea, redes sociales, pauta digital y blog), debido a que el $100 \%$ de los hoteles han utilizado por lo menos alguna de estas herramientas para promover su hotel, sin embargo, sólo son pocos los hoteles que establecen diferencias entre marketing digital y e-commerce, por lo que no cuentan con estrategias de marketing para aprovechar sus beneficios [6]. Por otra parte el sector hotelero de la Región Caribe Colombiana, realizó un diagnóstico de innovación y desarrollo tecnológico, con el que se indica que existe dependencia entre la innovación y su campo de gestión, donde la más baja participación la tienen los pequeños hoteles, sin embargo el tamaño no es determinante a la hora de innovar, puesto que todos le apuestan a capacitar a su capital humano en aspectos que contribuyan al desarrollo o mejoramiento de procesos, productos y servicios [7].

Finalmente a nivel local, se contó con el informe del estudio "Factores tecnológicos que influyen en la competitividad nacional e internacional del sector hotelero en la ciudad de Montería", en él se caracterizaron (4) niveles de uso de las TIC: 1 Básico, 2 Comercio electrónico y marketing digital, 3 Uso sistemático de las TIC en los procesos y 4 Uso estratégico de las TIC, lo que permitió medir el porcentaje de cumplimiento de cada uno de estos niveles por parte de los 40 hoteles encuestados (Básico 54\%, 2 Comercio electrónico y marketing digital 57\%, 3 Uso sistemático de las TIC $42 \%$ y 4 Uso estratégico de las TIC $32 \%$, donde el promedio de uso general de las TIC es del 46\% [1].

\section{MetodologíA}

Con el fin de indagar desde una perspectiva innovadora, cuáles son las TIC utilizadas en las MIPYMES del sector hotelero que contribuyen a su competitividad a nivel local, regional, nacional e internacional para estructurar un modelo para incorporarlas en sus procesos de negocio, se consultó sobre: el concepto de modelo, niveles o escalas de medición, modelos de incorporación o apropiación de las TIC, pasos para adoptar tecnología en las Pyme, MIPYMES, procesos de la gestión hotelera, TIC que proporciona beneficios competitivos a los hoteles, principales TIC que utiliza el turismo, soluciones tecnológicas en la industria del turismo, TIC más implementadas en los hoteles y que les ha ayudado a ser competitivos, competitividad, competitividad empresarial, y modelo para medir la competitividad empresarial.

Por otra parte, se describen las fases de construcción del Modelo para incorporar las TIC en las MIPYMES del sector hotelero, como el diseño, los instrumentos y los procedimientos realizados.

\section{B. Modelo}

Según el diccionario de la Real Academia Española un modelo es un arquetipo o punto de referencia para imitar o reproducir [8], sus funciones son: representar, explicar, guiar, motivar, predecir, evaluar y generar realidades [9], con el fin de representar la realidad en forma simplificada, se clasifican en modelo cualitativos, cuantitativos o mixtos, los cualitativos determinan de manera general las relaciones entre diferentes factores o componentes del sistema, los cuantitativos incluyen 
números y expresiones matemáticas [10] y los mixtos integran sistemáticamente los métodos cuantitativo y cualitativo [11].

\section{Niveles o escalas de medición}

La medición se refiere al proceso de asignar números o marcadores a objetos, personas, estados o hechos, teniendo en cuenta reglas específicas, con el fin de representar cantidades o cualidades de un atributo, a través de un sistema métrico específico [13]. Entre los que se encuentran: nominal, ordinal, de intervalos y de proporción [12], el nominal asigna números a objetos o fenómenos, sin valor numérico, el ordinal les da orden o prioridades a los datos (ascendente o descendente), escala de intervalos permite agrupar las mediciones por rangos iguales, escala de proporción o razón, muy similar a la escala de intervalos; con la diferencia que tiene un cero absoluto u origen.

\section{Modelos de incorporación o apropiación de las TIC}

Existen diferentes autores que han clasificado los modelos para la incorporación o apropiación de las TIC en las organizaciones ya sea por niveles, etapas, fases o pasos, [14] y [15], los clasifican por cuatro niveles, en el 1er nivel han considerado que la empresa tenga comunicaciones básicas como: teléfonos fijos o móviles; en el 2do nivel se refieren a tecnología informática básica como: un computador equipado con herramientas de ofimática básica ya sea licenciado o libre, para el 3er nivel se integran comunicaciones avanzadas como: navegación en internet que permita el uso de páginas web, correo y comercio electrónico, y la realización de video conferencias y el 4to nivel se ha tenido en cuenta tecnología informática avanzada como son las aplicaciones empresariales necesarias con funciones administrativas, financieras, comerciales, producción y capacitación.

Por otro lado [16] y [17], han clasificado el modelo por etapas, coincidiendo con [14] y [15], con una etapa inicial de tecnología básica en este caso computadoras e Internet, para la etapa dos se le suma aplicación de página web y software básico para crear información, automatizar procedimientos, incrementar la productividad de recursos humanos y reducir costos de las transacciones, una etapa tres en la que se cuenta con computadoras en red y cambios en el modelo de gestión (control y dirección) y una etapa cuatro donde las TIC se utilizan como elemento central para articular la organización a través de conexión de sistemas de información en las áreas con software diseñado a la medida y con computación en la nube, que por lo general son implementados por grandes empresas con intensiva ingeniería y servicios especializados.

[18] expresa el modelo en tres fases, en la primera la nombra Información (almacenamiento y procesamiento de datos), en la segunda Comunicación (donde se conforman redes de trabajo colaborativo y conocimiento para la toma de decisiones, la mejora del desempeño en procesos operativos y el favorecimiento de procesos de innovación empresarial y la tercera Flujos de trabajo (implementación de sistemas de información especializados para mejorar el desempeño de los procesos estratégicos de la compañía.

Por último [19] propone seis pasos, Iniciación donde se reconoce el impacto positivo de la tecnología en el funcionamiento de la organización; Inversión requiere planificar proyectos tecnológicos orientados a suplir necesidades y objetivos del negocio a corto, mediano y largo plazo; Adaptación que ajusta la tecnología a la cultura empresarial e integra el factor humano de la organización en su adopción; Apropiación se desarrollan nuevos usos y aplicaciones de las Tic para aumentar la competitividad de la empresa; Acción finalmente se toman acciones concretas, comenzando por buscar a un proveedor autorizado.

\section{E. MiPyMes}

Se entiende como unidad de explotación económica, realizada por personas naturales o jurídicas, en distintas actividades, que se clasifican según su planta de personal (trabajadores) y activos totales en términos de salarios mínimos mensuales legales vigentes SMLV en: medianas: planta de personal (51-200) y activos totales (5.001 - 15.000), pequeñas: Planta de personal (11-50), activos totales (501 - 5.001), Microempresa: Planta de personal no superior a los diez (10) trabajadores, activos totales inferior a (501) [20].

\section{F. Procesos de la gestión hotelera}

Procesos Estratégicos: responsables de emitir guías que respondan a necesidades y condicionantes de la sociedad, del mercado y de las empresas, Procesos Operativos o Claves: con los cuales el cliente percibirá y valorará la calidad, Procesos de Soporte o apoyo: responsables de proveer los recursos necesarios (personas, maquinaria y materia prima) y generar valor añadido deseado por los clientes [21]. Por su parte [22], enuncia que estos son: Proceso comercial: Captar, fidelizar y rentabilizar clientes. Proceso de Alojamiento: Registrar información formal y no formal de la interacción con clientes y ocupantes, identificando preferencias para brindar experiencias memorables en la estadía. Proceso de Alimentos y Bebidas. Dotación con dispositivos móviles para tomar pedidos. Proceso de Administración. Control remoto de la gestión del hotel, para administrar cargos, facturación y reportes.

\section{G. TIC que proporciona beneficios competitivos a los hoteles}

Entre las TIC más importantes por los beneficios competitivos al turismo y al sector hotelero según la revista [23] y [24] se tiene:

Property Management Systems (PMS) o "FRONT": sistema que brinda disponibilidad de habitaciones, reservas, cargos al huésped. Point of Sale (POS) o "puntos de venta": sistemas para procesar operaciones del huésped en centros de consumo como restaurantes, centros nocturnos, bares o centros de negocios. Back of the House Systems (BACK): son sistemas contables, administrativos y financieros para manejar las operaciones del negocio, provenientes de los sistemas PMS y POS. Central Reservation Systems (CRS): son centrales de voz que procesan reservaciones. Guest History $(\mathbf{G H})$ : es un registro histórico automatizado del uso de servicios turísticos de una persona, almacenando gustos y preferencias. Sistemas Globales de Distribución (GDS): es la búsqueda de información de vuelos, hospedaje, renta de autos, etc. Credit Card Key (CC Key): es la utilización del registro entre el huésped y su número de tarjeta de crédito para pagar consumos, como llave de la habitación. Yield Management (YM) o Revenue Management: es un sistema que analiza las operaciones para controlar el precio y oferta de los servicios basándose en la demanda e inventario, Cloud Computing: es 
el acceso a los servicios computacionales como redes, servidores, almacenamiento, aplicaciones y servicios por demanda, Redes Sociales: son plataformas digitales donde los usuarios intercambian contenidos, bienes y servicios, ECommerce: es la distribución, venta, compra, marketing y suministro de información de productos o servicios a través de Internet, Customer Relationship Management - Gestión de Relaciones con el Cliente (CRM). Permite que se mejoren las relaciones entre la empresa y sus clientes por medio de la comunicación. Enterprise Resource Planning - Planificación de Recursos Empresariales (ERP). Permite la administración de los procesos operativos, al integrar varias funcionalidades de gestión en un único sistema.

\section{H. Competitividad}

Aptitudes, habilidades y capacidades de una empresa o país, para vender aquello que es producido (bienes y/o servicios), con patrones de calidad específicos, requeridos por el mercado, utilizando recursos de manera eficiente (alcanzar las metas al menor costo posible) y efectiva (alcanzar los objetivos correctos). Capacidad de enfrentar la competencia a nivel mundial y alcanzar en forma sostenida altas tasas de crecimiento del Producto Interno Bruto (PIB) per cápita, para lograr objetivos en política económica (ingresos y empleo), habilidades de crear, producir, distribuir y/o servir productos en el comercio internacional, al tiempo que mayores retornos, para sostener y expandir su participación en mercados internacionales y elevar el nivel de vida de su población [25].

\section{Competitividad empresarial}

Según [26], la competitividad empresarial se refiere a la capacidad productiva que se tiene para diseñar, desarrollar, producir y colocar productos en mercados internacionales, al tiempo que sus competidores, respondiendo de manera ventajosa, o generando mayor valor agregado que sus competidores

\section{J. Modelo para medir la competitividad empresarial.}

De acuerdo con [27], existen varios modelos para medir la competitividad, entre ellos se tiene:

A nivel empresarial el modelo matemático para determinar la competitividad de las PYMES y el modelo de competitividad de la Universidad Manuela Beltrán, el primero considera medir variables como tecnología, administración, producción, recurso humano y el entorno empresarial, el segundo afirma que se debe medir la capacidad de gestión de la gerencia por medio de los resultados y el posicionamiento de las organizaciones, expresando que estos son el reflejo de sus gerentes.

A nivel país se encuentran el Índice Global de Competitividad desarrollado por el Foro Económico Mundial, quienes expresan la competitividad como la capacidad de sostener económicamente la nación y brindar prosperidad a sus habitantes, parecido con lo que propone el Centro de Investigaciones para el Desarrollo, quienes trabajan con los siguientes indicadores: la competitividad en el tiempo (ICDE), el crecimiento en el mercado y en la calidad de vida (ICDR).

Entre otros modelos están el del Instituto para el Desarrollo de la Gerencia que se refiere a la capacidad para sostener la competitividad de las empresas, o el de la Universidad Autónoma de México donde la competitividad regional se refiere a la producción, valor agregado y utilidades regionales y el del Centro Regional de Estudios Cafeteros y Empresariales que ha considerado cinco dimensiones: la social (capital humano y social del departamento), la estructural (crecimiento económico regional y cambios estructurales determinantes en el crecimiento), la institucional (identificación de instituciones en los municipios), empresarial (aplicación de una encuesta a empresarios) y la regional.

\section{K. Narrativa del Diseño}

Etapa 1. Enfoque mixto con alcance exploratorio, mixto recolecta, analiza y vincula datos cuantitativos y cualitativos en un mismo estudio para responder a un planteamiento del problema, [28], exploratorio no requiere establecer relaciones entre dos o más variables, tampoco intenta predecir un dato o valor en una o más variables que se vayan a medir u observar, [28].

\section{Narrativa de los Instrumentos}

Etapa 2. La metodología para la revisión bibliográfica fue la búsqueda normal y avanzada en bases de datos, revistas, recursos de acceso abierto, páginas web de instituciones, para identificar estudios previos referentes al tema con no más de diez años de antigüedad como lo recomienda [28] y [29].

\section{Narrativa del Procedimiento}

Etapa 3. A partir de la revisión bibliográfica se identificaron las TIC que se utilizan en los diferentes procesos de las organizaciones hoteleras y los beneficios competitivos que cada una de estas tributan a dicho sector, posteriormente se caracterizaron en una escala ordinal en cuatro niveles de menor a mayor según el grado de complejidad de implementación y los beneficios que estas aportan al fortalecimiento de la competitividad del sector hotelero, en cada nivel se describe las aplicaciones tecnológicas y los beneficios que estas aportan, de acuerdo con [12], una escala ordinal tiene como propósito dar orden a los datos de forma ascendente o descendente.

Para estructurar el modelo, se tuvo en cuenta diseños previos de incorporación o apropiación de las TIC propuestos por [14], [15], [16], [17], [18] y [19] y la caracterización de las TIC realizada previamente en este estudio, lo que permitió definir los cuatro (4) niveles que conforman el modelo propuesto.

\section{RESUltados}

El modelo se encuentra estructurado en cuatro niveles (Ver Fig. 1) que le permite a una organización hotelera implementar las TIC a corto, mediano y largo plazo, no solo para desarrollar tareas simples para ser más eficientes sino, avanzar de forma gradual y progresiva a un nivel intensivo y estratégico en tareas más complejas facilitando el control de los procesos operacionales, la fidelización de los clientes, la promoción de sus servicios a nivel global y potenciar su competitividad empresarial. 


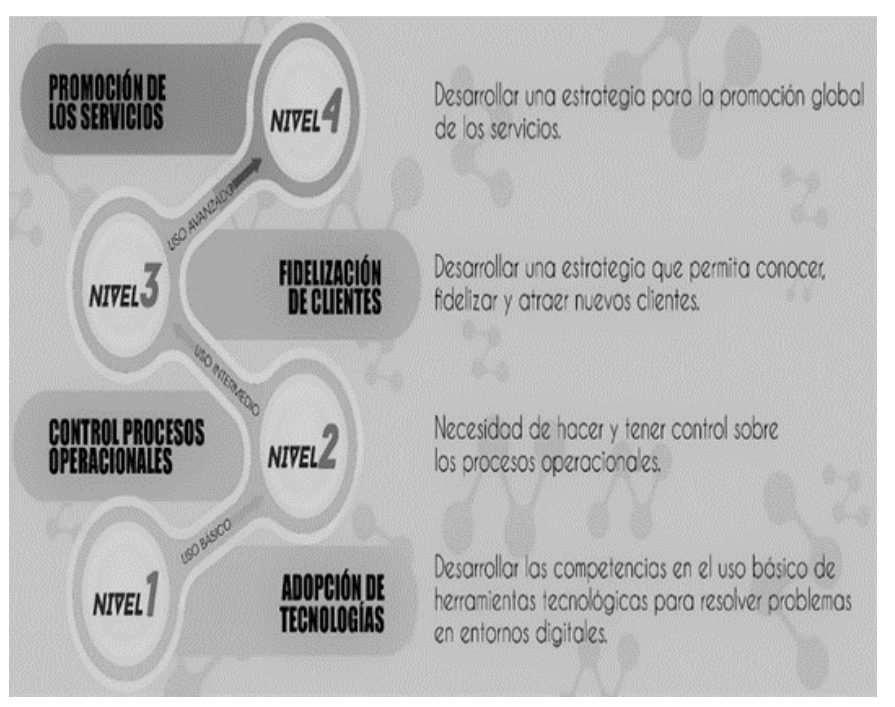

Fig 1. Modelo para incorporar las TIC en las MIPYMES del sector hotelero

\section{A. Nivel 1 Adopción de tecnologías}

Es el nivel más básico, se reconoce la importancia de capacitar y formar al talento humano para desarrollar las competencias en el uso de herramientas tecnológicas para resolver problemas en entornos digitales (Ver tabla I); son un elemento primordial para la competitividad, mejoran o agilizan los procesos y aumentan la productividad, permiten recopilar, almacenar, tratar y analizar la información para apoyar la toma de decisión, mejorando los procesos de comunicación con los clientes y proveedores [35].

TABLA I

CARACTERIZACIÓN NIVEl I ADOPCIÓN DE TECNOLOGÍAS

\begin{tabular}{|c|c|}
\hline $\begin{array}{l}\text { Aplicación } \\
\text { de las } \\
\text { tecnologías }\end{array}$ & Beneficios \\
\hline $\begin{array}{l}\text { Informática } \\
\text { básica }\end{array}$ & $\begin{array}{l}\text { - Desarrollar competencias digitales } \\
\text { - Resolver problemas en entornos digitales }\end{array}$ \\
\hline Ofimática & $\begin{array}{l}\text { - Optimizar, automatizar y mejorar los } \\
\text { procedimientos o tareas relacionadas con funciones } \\
\text { de oficina. } \\
\text { - Crear, manipular, transmitir, almacenar y consultar } \\
\text { información de forma ágil y oportuna }\end{array}$ \\
\hline $\begin{array}{l}\text { Trabajo } \\
\text { colaborativo } \\
\text { en línea }\end{array}$ & $\begin{array}{l}\text { - Se eliminan barrera de tiempo y espacio } \\
\text { - Comunicación más eficaz y directa } \\
\text { - Facilita la dirección, control y supervisión del } \\
\text { trabajo } \\
\text { - Promueve el proceso de enseñanza - aprendizaje } \\
\text { - Trabajo multidisciplinar } \\
\text { - Mejores resultados y toma de decisiones } \\
\text { - Permite realizar procesos en paralelo } \\
\text { - Seguimiento de la información en tiempo real }\end{array}$ \\
\hline $\begin{array}{l}\text { Búsqueda de } \\
\text { información } \\
\text { documental }\end{array}$ & $\begin{array}{l}\text { - Significativo número de fuentes de consulta } \\
\text { - Información actualizada } \\
\text { - Reducción de tiempo y costo } \\
\text { - Es posible encontrar muchos puntos de vista } \\
\text { diferentes }\end{array}$ \\
\hline $\begin{array}{l}\text { Herramientas } \\
\text { de } \\
\text { comunicación }\end{array}$ & $\begin{array}{l}\text { - Económico } \\
\text { - Ágil } \\
\text { - Versátil } \\
\text { - Personalizable } \\
\text { - Permite compartir información en tiempo real }\end{array}$ \\
\hline
\end{tabular}

- Interactivo

\section{B. Nivel 2 Control de procesos operacionales}

Se reconoce la necesidad de hacer y tener control sobre los procesos operacionales apoyado con herramientas tecnológicas (Ver tabla II), con el propósito de garantizar un servicio eficiente, personalizado, confiable y de calidad, cumpliendo con las expectativas de todos los interesados, orientado a la gestión de calidad y sostenibilidad turística; Con el fin de atender una demanda turística cada vez más exigente, mejor informada y las necesidades de mejoramiento de la calidad de la oferta turística bajo un enfoque de turismo sostenible, [36]; La calidad del servicio en la hotelería contribuye al posicionamiento del establecimiento lo cual genera confiabilidad en los viajeros, promoviendo un grado de satisfacción que redundará en una buena imagen, [37].

TABLA II

CARACTERIZACIÓN NIVEL II CONTROL DE PROCESOS OPERACIONALES

\begin{tabular}{|c|c|}
\hline $\begin{array}{l}\text { Aplicación de } \\
\text { las tecnologías }\end{array}$ & Beneficios \\
\hline $\begin{array}{l}\text { Sistema de } \\
\text { Gestión } \\
\text { Hotelera } \\
\text { (PMS) }\end{array}$ & $\begin{array}{l}\text { - Dejar de pagar por las reservas propias } \\
\text { - Optimización de recursos y de tiempo } \\
\text { - Actualización automatizada e inmediata de todos los } \\
\text { canales on-line } \\
\text { - Gestión desde cualquier lugar } \\
\text { - Mayor conocimiento del huésped } \\
\text { - Permite que la oferta llegue a más clientes } \\
\text { - Mejor servicio y atención al huésped }\end{array}$ \\
\hline $\begin{array}{l}\text { Terminales } \\
\text { Punto de Venta } \\
\text { (TPV) }\end{array}$ & $\begin{array}{l}\text { - Facilita el pago de los clientes mediante tarjetas de } \\
\text { crédito y débito } \\
\text { - Reducción en manejo de efectivo, se traduce en mayor } \\
\text { seguridad } \\
\text { - Evita hacer tantos cortes de caja al día ahorrando } \\
\text { tiempo } \\
\text { - Se cuenta con un registro de todos los movimientos } \\
\text { financieros } \\
\text { - Ajuste o actualización de precios } \\
\text { - Control de ingresos } \\
\text { - Control de inventario }\end{array}$ \\
\hline $\begin{array}{l}\text { Sistema } \\
\text { Central de } \\
\text { Reservas } \\
\text { (CRS) } \\
\text { Sistema Global } \\
\text { de Distribución } \\
\text { (GDS) }\end{array}$ & $\begin{array}{l}\text { - Sin límite de tiempo } 24 * 7 \text { los } 365 \text { días del año } \\
\text { - Reduce el trabajo manual } \\
\text { - Descuentos y códigos promocionales } \\
\text { - Pago online } \\
\text { - Mejora la marca, gana reconocimiento } \\
\text { - Mejora la eficiencia } \\
\text { - Aumento de la calidad del servicio } \\
\text { - Mayor personalización de la oferta }\end{array}$ \\
\hline $\begin{array}{l}\text { Planificación } \\
\text { de Recursos } \\
\text { Empresariales } \\
\text { (ERP) }\end{array}$ & $\begin{array}{l}\text { - Incremento del control de la empresa } \\
\text { - Visión global } \\
\text { - Integración de los procesos claves del negocio } \\
\text { - Conectividad con sistemas externos } \\
\text { - Estandarización } \\
\text { - Mejora la eficiencia y capacidad operativa } \\
\text { - Información Integrada } \\
\text { - Ahorro de costos, más competitividad }\end{array}$ \\
\hline $\begin{array}{l}\text { Sistema de } \\
\text { Administración } \\
\text { de Inventario } \\
(\mathrm{WMS}))\end{array}$ & $\begin{array}{l}\text { - Aumenta la productividad de un trabajador en el } \\
\text { almacén } \\
\text { - Incrementa la exactitud de la existencia } \\
\text { - Reduce costos y tiempo } \\
\text { - Maximiza la estrategia de distribución }\end{array}$ \\
\hline $\begin{array}{l}\text { Sistemas de } \\
\text { Información }\end{array}$ & $\begin{array}{l}\text { - Permite comparar resultados con los objetivos } \\
\text { programados }\end{array}$ \\
\hline
\end{tabular}


Gerencial - Brinda una amplia y completa gama de información

(MIS) - Permite seleccionar, combinar y filtrar información según requerimiento

- Generar reportes estadísticos

- Permite un control exacto y oportuno de la información

- Genera un sistema de alertas

- Generar documentos de orden legal

- Mejora la efectividad en la toma de decisión

- Disponibilidad de mayor y mejor información para los usuarios

- Disminuye errores, tiempo y recursos

\section{Nivel 3 Fidelización de clientes}

La organización reconoce el efecto sinérgico de desarrollar una estrategia que permita atraer, mantener y desarrollar las relaciones con los clientes con el apoyo de herramientas tecnológicas (Ver tabla III); para ello se deben aplicar políticas de fidelización, evitando la fuga de clientes hacia la competencia. [38], el uso eficiente y eficaz de estas herramientas, contribuirá positivamente a incrementar el nivel de competitividad del hotel, puesto que se cuenta con mayor y mejor información para la toma de decisión.

TABLA III

CARACTERIZACIÓN NIVEL III FIDELIZACión De CLIENTES

\begin{tabular}{|c|c|}
\hline $\begin{array}{l}\text { Aplicación } \\
\text { de las } \\
\text { tecnologías }\end{array}$ & Beneficios \\
\hline $\begin{array}{l}\text { Gestión de } \\
\text { relaciones } \\
\text { con el cliente } \\
(\mathrm{CRM})\end{array}$ & $\begin{array}{l}\text { - Identifica nuevas oportunidades de venta } \\
\text { - Ayuda a mejorar la reputación } \\
\text { - Mejor conocimiento de los clientes y prospectos } \\
\text { - Conocer específicamente que necesitan los clientes para } \\
\text { ofrecérselo } \\
\text { - Obtener información actualizada en tiempo real de los } \\
\text { clientes } \\
\text { - Ofrecer soluciones individualizadas a los clientes } \\
\text { - Predecir comportamientos de los clientes. }\end{array}$ \\
\hline Big Data & $\begin{array}{l}\text { - Detección de tendencias } \\
\text { - Conocimiento de clientes } \\
\text { - Desarrollo de nuevos productos o servicios } \\
\text { - Análisis predictivo } \\
\text { - Optimización de procesos } \\
\text { - Atracción y fidelización de clientes } \\
\text { - Planes estratégicos inteligentes de marketing } \\
\text { - Mejora en la eficiencia y costos } \\
\text { - Retroalimentación en tiempo real }\end{array}$ \\
\hline $\begin{array}{l}\text { Computación } \\
\text { en la nube }\end{array}$ & $\begin{array}{l}\text { - Capacidad de almacenamiento } \\
\text { - Copias de seguridad } \\
\text { - Escalabilidad } \\
\text { - Movilidad } \\
\text { - Optimización del uso de los recursos } \\
\text { - Reducción de costos } \\
\text { - Reducción del timer to market } \\
\text { - Respecto al medio ambiente } \\
\text { - Disponibilidad } \\
\text { - Abstracción de la parte técnica }\end{array}$ \\
\hline $\begin{array}{l}\text { Internet de } \\
\text { las cosas } \\
\text { (IoT) }\end{array}$ & $\begin{array}{l}\text { - Velocidad de análisis de datos } \\
\text { - Facilidad de seguimiento } \\
\text { - Ahorro en tiempo en las tareas de monitorización } \\
\text { - Ahorro en dinero, podría reemplazar a los humanos que } \\
\text { están a cargo de monitorizar y gestionar los suministros }\end{array}$ \\
\hline
\end{tabular}

\section{Nivel 4 Promoción de los servicios}

El hotel desarrolla una estrategia para la promoción global de los servicios con el apoyo de herramientas tecnológicas (Ver tabla IV), lo que le permite ampliar sus fronteras con el propósito de expandirse a nuevos mercados para atraer nuevos clientes.

TABLA IV

Caracterización Nivel 4 Promoción De Los Servicios

\begin{tabular}{ll}
\hline \hline $\begin{array}{l}\text { Aplicación } \\
\text { de las } \\
\text { tecnologías }\end{array}$ & Beneficios \\
\hline Comercio & -En línea a través de Internet \\
electrónico & -Sin límite de tiempo $24^{*} 7$ los 365 días del año \\
& - Global, no tiene limitaciones físicas \\
& -Plataforma única y uniforme para el intercambio de \\
& información \\
& -Relaciones comerciales de extremo a extremo \\
& -Sin costos fijos \\
& -Ahorro de tiempo, evita colas \\
& -La comunicación es interactiva y bidireccional, el usuario \\
interactúa \\
digital) & -Los consumidores llegan a través de motores de \\
& búsqueda, indicaciones y social media \\
& -Se puede medir y analizar el impacto \\
& -Logra entretener, educar y aportar contenido de valor \\
& para el cliente \\
& -Busca atraer a los clientes de manera voluntaria y no de \\
& forma intrusiva \\
& - Concentra sus esfuerzos en deleitar al cliente siempre \\
& -Llegar a 1000 personas tiene un costo que oscila entre 1 y \\
& 10 USD \\
\hline \hline & \\
\hline
\end{tabular}

\section{CONCLUSION}

La caracterización de las TIC permitió clasificarlas en cuatro niveles de menor a mayor grado de complejidad de implementación y beneficios competitivos que aportan al sector hotelero, insumo principal para la estructuración del modelo.

Con la estructuración del modelo se espera que los hoteles inicien o continúen su proceso de transformación digital para beneficiarse de las ventajas competitivas que proporcionan las herramientas tecnológicas propuestas.

El modelo se convierte en una hoja de ruta hacia la transformación digital de dicho sector, llevándolos desde un nivel de uso básico de las TIC hasta llegar a un nivel intensivo y estratégico de uso de estas.

Aunque el modelo fue concebido para ser implementado en las organizaciones del sector hotelero de la ciudad de Montería, este puede ser implementado por cualquier hotel sin importar su tamaño (micro, pequeña, mediana y grande) y su ubicación geográfica, dado a que en su estructuración se tuvieron en cuenta los procesos que se desarrollan en este tipo de organizaciones a nivel local, regional, nacional e internacional, las aplicaciones tecnológicas (TIC) propuestas abarcan todos estos procesos.

El modelo puede ser adaptado a cualquier otro sector económico, para ello se deben identificar las TIC que conformarían el nivel 2 control de los procesos operacionales y sus beneficios, dado a que son distintas para cada sector.

No necesariamente un hotel debe implementar los cuatro niveles, se debe inicialmente hacer un diagnóstico para determinar el nivel de uso y de acuerdo con los resultados determinar el nivel o si requiere implementar parte de uno o más 
niveles para avanzar luego al siguiente nivel. En este sentido el modelo es flexible, de acuerdo con la necesidad de cada hotel, se seleccionan e implementan las TIC que se requieran según la lista propuestas u otras que puedan ser adicionadas según como avance el desarrollo tecnológico, por lo que el modelo no perderá vigencia alguna en el tiempo.

El modelo se alinea con las políticas del Ministerio de las TIC - MinTIC en el sentido que buscan que los empresarios del sector hotelero se apropien del uso de las tecnologías para que sean más competitivos.Appendixes, if needed, appear before the acknowledgment.

\section{REFERENCIAS}

[1]. Y. Arcia, J. Castillo, E. Reals, C. Hernández, J. Guerra, A. Lozano, E. Esteban y M. Arteaga, "Factores tecnológicos que influyen en la competitividad nacional e internacional del sector hotelero en la ciudad de Montería," Montería, 2017.

[2]. Fundetec - ONTSI, "Informe ePyme 2014. Análisis de implantación de las TIC en la pyme española," 2014. [En línea]. Available: http://www.ipyme.org/publicaciones/informe-epyme-2014.pdf.

[3]. R. Gyorgy, "Introducción y difusión de las nuevas tecnologías de la información en el sector hotelero,” 2013. [En línea]. Available: http://repositori.uji.es/xmlui/bitstream/handle/10234/129928/TFG_ 2014_GyorgyR.pdf?sequence=1.

[4]. M. Ruiz, I. Gil y B. Moliner, "Las tecnologías que implementan los hoteles y las tecnologías que gustan a los huéspedes," 2013. [En línea]. $\quad$ Available: http://www.aecit.org/jornal/index.php/AECIT/article/viewFile/144/ 126.

[5]. S. Alonso, F. Lanuza y N. Golovina, "Tecnología de la Información y Comunicación (TIC) como estrategias de marketing y su contribución a la competitividad del sector turístico hotelero de la Ciudad de Estelí, Nicaragua, en el I semestre del 2016," 2016. [En línea]. Available: http://www.camjol.info/index.php/FAREM/article/view/2972/2721

[6]. A. Bucheli, A. Vaegas, D. Restrepo, H. Iván, E. Martínez y S. Romero, "Estudio de la utilización del marketing digital en el sector hotelero de la ciudad de Bogotá," 2013. [En línea]. Available: http://www.utadeo.edu.co/files/collections/documents/field_attach ed_file/cartilla.pdf?width $=740$ \&height $=780$ \&inline $=$ true.

[7]. J. Jiménez, J. Mojica, H. Hernández y D. Cardona, "Diagnóstico de la Innovación y Desarrollo Tecnológico en el Sector Hotelero de la Región Caribe Colombiana," Información Tecnológica, vol. Vol. $29, \mathrm{n}^{\mathrm{o}} \quad 5$. pp. 157-164.2018. DOI: 10.4067/S071807642018000500157

[8]. Real Academia Española, "Diccionario de la lengua española," 2017. [En línea]. Available: http://www.rae.es/.

[9]. L. Sesento, "Modelo sistemático basado en competencias para instituciones educativas públicas," 2008. [En línea]. Available: http://www.eumed.net/tesis-doctorales/2012/lsg/indice.htm.

[10]. FAO, "Modelos y su uso," s. f.. [En línea]. Available: http://www.fao.org/docrep/w7452s/w7452s01.htm.

[11]. R. H. Sampieri, C. F. Collado y M. d. P. B. Lucio, Metodología de la investigación, México: McGrawHill, 2010.

[12]. C. Bernal, Metodología de la investigación, México: Pearson, 2010.

[13]. J. C. Padilla, "Escala de medición," Paradigma, vol. 2, n² 2, pp. 104$125,2007$.

[14]. MinComunicaciones, "Políticas para la promoción en el acceso y uso de TIC en micro, pequeñas y medianas empresas colombianas," 2009. [En línea]. Available: https://spi.dnp.gov.co/App_Themes/SeguimientoProyectos/Resum enEjecutivo/090518\%20\%20Politica\%20Uso\%20de\%20TIC\%20n\%20mipymes\%20$\% 20$ MinComunicaciones.pdf.

[15]. V. K. "Small and Medium Enterprises and ICT," 2007. [En línea]. Available: http://www.unapcict.org/ecohub/resources/small-andmedium-enterprises-and-ict.
[16]. M. Slusarczyk Antosz, J. Pozo Rodríguez y L. Perurena Cancio, "Estudio de aplicación de las TIC en las MIPYMES," 2014. [En línea]. Available: http://www.3ciencias.com/wpcontent/uploads/2015/02/ESTUDIO-DE-APLICACI\%C3\%93NDE-LAS-TIC-EN-LAS-PYMES.pdf.

[17]. CEPAL, "Importancia de las TIC en la mejora competitiva de las PyMe," 2013. [En línea]. Available: http://www.imaginar.org/taller/ticyproductividad/docs/P11_godana_stojkovic.pdf.

[18]. J. Chaverra y A. Arias, "El papel de las TIC en el entorno organizacional de las PYMES," 2012. [En línea]. Available: https://dialnet.unirioja.es/servlet/articulo?codigo $=4521389 \mathrm{https}: / / \mathrm{d}$ ialnet.unirioja.es/servlet/articulo?codigo $=4521389$.

[19]. Cade Soluciones, "5 pasos para adoptar tecnología en tu PyME," 2013. [En línea]. Available: https://cadesoluciones.wordpress.com/2013/08/07/5-pasos-paraadoptar-tecnologia-en-tu-pyme/.

[20]. Ley 590, 2010.

[21]. M. Domínguez, "Gestión por proceso en el área de alojamiento en las instalaciones hoteleras," s.f. [En línea]. Available: https://www.gestiopolis.com/gestion-por-proceso-en-el-area-dealojamiento-hotelero-cuba/.

[22]. CSI S.A., "Los principales procesos de un hotel y sus objetivos," s.f. [En línea]. Available: http://www.csisasoluciones.com/noticiashoteles/los-principales-procesos-de-un-hotel-y-sus-objetivos.html.

[23]. Turismo \& Tecnología, "Uso de TICs en Turismo, nuevas tecnologías al servicio del viajero," 2014. [En línea]. Available: http://www.turismoytecnologia.com/todos-los-articulos-detecnologia/item/3617-uso-de-tics-en-turismo-nuevas-tecnologiasal-servicio-del-viajero.

[24]. Fundación Orange, "La transformación digital en el sector turístico," 2016. [En línea]. Available: http://www.fundacionorange.es/wpcontent/uploads/2016/05/eE_La_transformacion_digital_del_sector -turistico.pdf.

[25]. Ministerio de Economía, "Competitividad: marco conceptual y análisis sectorial para la provincia de Buenos Aires,” 2006. [En línea].

Available: http://www.ec.gba.gov.ar/areas/estudios_proyecciones/Archivos/C uaderno74.pdf.

[26]. A. Cabrera, P. López y C. Ramírez, "La competitividad empresarial: un marco conceptual para su estudio,” 2011. [En línea]. Available: https://www.ucentral.edu.co/images/documentos/editorial/2015_co mpetitividad_empresarial_001.pdf.

[27]. L. Castaño y A. Gutiérrez, "Propuesta para determinar la competitividad en las empresas del sector comercial del área metropolitana centro occidente AMCO,” 2011. [En línea]. Available:

http://repositorio.utp.edu.co/dspace/bitstream/handle/11059/2041/6 58406C346.pdf;jsessionid=438332C2F8717D35FED991677BB9A F2E? sequence $=1$.

[28]. R. Hernández, C. Fernández y P. Baptista, Metodología de la investigación, México: Mc Graw Hill, 2006.

[29]. E. Esteban, "Importancia del desarrollo de habilidades académicas como estrategia para el inicio de la formulación y evaluación de proyectos tecnológicos," 2016. DOI: 10.16925/greylit.1848.

[30]. S. Maldonado, L. Méndez y J. Peña, "Manual práctico para el diseño de la Escala Likert," 2007. [En línea]. Available: https://dialnet.unirioja.es/descarga/articulo/4953744.pdf.

[31]. R. Hernández, C. Fernández y P. Baptista, Metodología de la investigación, México: McGrawHill, 2010.

[32]. M. Saavedra y B. Tapia, "El uso de las tecnologías de información y comunicación TIC en las micro, pequeñas y medianas empresas (MIPyME) industriales mexicanas,” 2013. [En línea]. Available: https://dialnet.unirioja.es/descarga/articulo/4334695.pdf.

[33]. CPTUR, "Índice de competitividad turística regional de Colombia ICTRC 2017,” 2017. [En línea]. Available: http://www.cptur.org/Publicaciones/o0GVkLQsHFpRqCD.

[34]. Andalucía Digital, "Ventajas de las TIC para las empresas," s.f. [En línea]. Available: https://www.blog.andaluciaesdigital.es/ventajasde-las-tics-para-las-empresas/.

[35]. M. Rios, J. Toledo, O. Campos y A. Alejos, "Nivel de integración de las TICS en las MIPYMS, un análisis cualitativo," 2009. [En línea].

Available: 
http://admon.itc.mx/ojs/index.php/\%20panorama/article/viewFile/1 $50 / 161$.

[36]. [ICONTEC, "Norma Técnica Sectorial Colombiana NTS-TS 002," 2006. [En línea]. Available: http://www.minambiente.gov.co/images/normativa/Otros/NTC/200 6/NTSTS_002_2006.pdf.

[37]. G. Janusz y P. Bajdor, "Towards to Sustainable Tourism Framework, Activities and Dimensions," 2013. [En línea]. Available: https://ac.els-cdn.com/S2212567113001706/1-s2.0S2212567113001706-main.pdf?_tid=51aebc90-0f26-409a-9bf64bbe57665d38\&acdnat=1530548717_8fe8d246c012e58fad3df7e1 94536a0d.

[38]. L. Agüero, "Estrategia de fidelización de clientes,” 2013. [En línea]. Available: https://repositorio.unican.es/xmlui/bitstream/handle/10902/4474/\% 5B2\%5D\%20Ag\%C3\%BCero\%20Cobo\%20L.pdf.

[39]. Hotelerum, "5 estrategias eficacs de marketing para los hoteles de hoy," 2015. [En línea]. Available: https://www.hotelerum.com/5estrategias-de-marketing-para-hoteles/.

[40]. D. Solorzano, L. Ascanio, P. Ruiz y M. Neira, "Sistema de Gestión de Calidad en el Sector Hotelero," 2016. [En línea]. Available: http://www.fesc.edu.co/Revistas/OJS/index.php/convicciones/articl e/view/167/221.

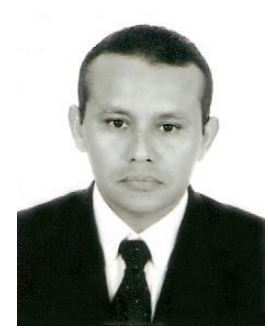

Máximo Miguel Arteaga Martínez Ingeniero informático Fundación Universitaria Católica del Norte, Magister en Gestión de Tecnologías de la información Universidad Cooperativa de Colombia. Docente programa de Ingeniería de sistemas Corporación Universitaria Remington.

ORCID: https://orcid.org/0000-0002-0431-6315

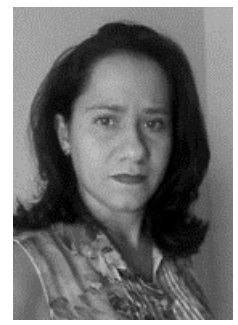

Edna Margarita Esteban Regino Ingeniera de sistemas de información Universidad del Sinú, Especialista en Ingeniería de software Universidad distrital Francisco José de Caldas, Magister en Tecnología Educativa y Medios Innovadores para la Educación. Docente programa de Ingeniería de sistemas Universidad Cooperativa de Colombia.

ORCID: https://orcid.org/0000-0001-7790-4532

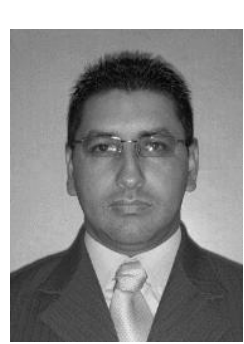

Miguel Antonio Ojeda Enríquez Ingeniero de sistemas, Universidad Autónoma de Colombia; Especialista en Teleinformática, Universidad distrital Francisco José de Caldas; Magister en Ingeniería de Software. Docente del programa de Ingeniería de sistemas Universidad Cooperativa de Colombia.

ORCID: https://orcid.org/0000-0002-8774-4314 